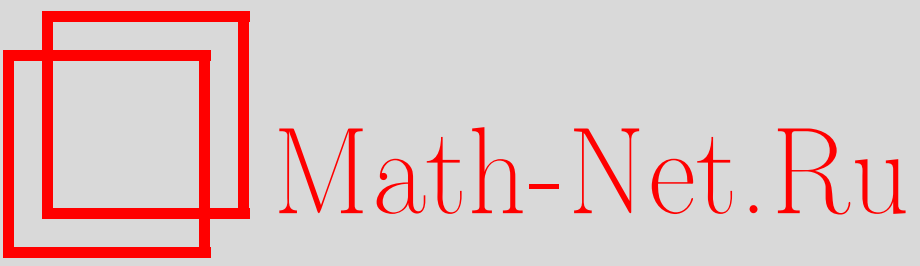

Р. Габасов, Н. М. Дмитрук, Ф. М. Кириллова, О проблеме оптимального управления динамическими системами в реальном времени, Итоги науки и техн. Сер. Соврем. мат. и ее прил. Темат. обз., 2020, том 183, 98-112

DOI: https://doi.org/10.36535/0233-6723-2020-183-98-112

Использование Общероссийского математического портала Math-Net.Ru подразумевает, что вы прочитали и согласны с пользовательским соглашением

http://www.mathnet.ru/rus/agreement

Параметры загрузки:

IP: 52.90 .164 .192

26 апреля 2023 г., 14:06:58 


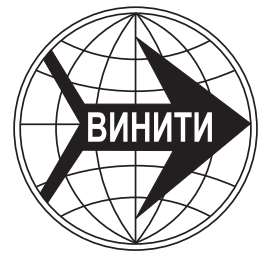

ИТОГИ НАУКИ И ТЕХНИКИ.

Современная математика и ее приложения.

Тематические обзоры.

Том 183 (2020). С. 98-112

DOI: $10.36535 / 0233-6723-2020-183-98-112$

УДК $519.714,517.977$

\title{
О ПРОБЛЕМЕ ОПТИМАЛЬНОГО УПРАВЛЕНИЯ ДИНАМИЧЕСКИМИ СИСТЕМАМИ В РЕАЛЬНОМ ВРЕМЕНИ
}

\author{
(c) 2020 г. \\ Р. ГАБАСОВ, Н. М. ДМИТРУК, Ф. М. КИРИЛЛОВА
}

\begin{abstract}
АннотАция. В работе приводится обзор результатов по проблеме оптимального управления в реальном времени линейными системами, полученных минской школой по математическим методам оптимального управления. Рассматриваются задачи оптимального управления динамическими объектами по их детерминированным математическим моделям и совершенным измерениям состояний, объектами с возмущениями и несовершенными измерениями наблюдаемых входных и выходных сигналов, задачи оптимального децентрализованного управления группами взаимосвязанных динамических объектов, а также применение задач оптимального управления и принципа управления в реальном времени к решению проблем стабилизации.
\end{abstract}

Ключевые слова: оптимальное управление, управление в реальном времени, неопределенность, несовершенные измерения, децентрализованное управление, стабилизация, алгоритм.

\section{ON THE PROBLEM OF OPTIMAL CONTROL OF DYNAMIC SYSTEMS IN REAL TIME}

\author{
(c) 2020 R. GABASOV, N. M. DMITRUK, F. M. KIRILLOVA
}

\begin{abstract}
This paper is a review of the results on the problem of optimal control in real time for linear systems, obtained by the Minsk school by mathematical methods of optimal control. We consider optimal control problems for dynamic objects, their deterministic mathematical models and perfect measurements of states, objects with disturbances and imperfect measurements of the observed input and output signals, the problem of optimal decentralized control of groups of interconnected dynamic objects, and application of real-time optimal control problems and the control principle to solving stabilization problems.
\end{abstract}

Keywords and phrases: optimal control, real-time control, uncertainty, imperfect measurements, decentralized control, stabilization, algorithm.

AMS Subject Classification: 37C75, 93D15, 93C05, 49J15

1. Введение. Управление в реальном времени - один из современных подходов к управлению динамическими объектами по принципу замкнутого контура. При управлении в реальном времени обратные связи (позиционные решения) не строятся, а необходимые для управления их текущие значения формируются в реальном времени по ходу каждого конкретного процесса. Основу подхода составляют алгоритмы работы оптимальных регуляторов, которые в каждый момент времени решают задачу оптимального управления, сформулированную на основе той информации, что доступна к текущему моменту времени (текущей позиции процесса управления). Значение оптимального программного управления этой задачи в текущий момент времени есть значение обратной связи на текущей позиции. Оно подается на вход объекта управления до поступления следующего измерения, порождая таким образом реализацию обратной связи в конкретном процессе управления. 
Настоящий обзор посвящен применению принципа управления в реальном времени и особенностям построения алгоритмов работы оптимальных регуляторов при реализации оптимальных обратных связей в задачах оптимального управления по детерминированным математическим моделям и совершенным измерениям состояний динамических объектов (раздел 2), по математическим моделям с возмущениями и несовершенным измерениям (раздел 3 ), а также в актуальных для приложений задачах управления группами динамических объектов (раздел 4) и задачах стабилизации (раздел 5).

2. Оптимальное управление динамическим объектом по совершенным измерениям его состояний. На промежутке времени $T=\left[t_{*}, t^{*}\right]$ рассмотрим задачу оптимального управления динамическим объектом, для которого принята линейная нестационарная детерминированная модель

$$
\begin{array}{ll}
c^{\prime} x\left(t^{*}\right) \rightarrow \max , & \\
\dot{x}=A(t) x+B(t) u, & x\left(t_{*}\right)=x_{0}, \\
x\left(t^{*}\right) \in X^{*}, & u(t) \in U, \quad t \in T .
\end{array}
$$

Здесь $x=x(t) \in \mathbb{R}^{n}, u=u(t) \in \mathbb{R}^{r}$ - состояние и управляющее воздействие в момент времени $t$; $A(t) \in \mathbb{R}^{n \times n}, B(t) \in \mathbb{R}^{n \times r}, t \in T,-$ кусочно непрерывные матричные функции; $X^{*}=\left\{x \in \mathbb{R}^{n}\right.$ : $\left.g_{*} \leqslant H x \leqslant g^{*}\right\}$ - терминальное множество, $g_{*}, g^{*} \in \mathbb{R}^{m}, H \in \mathbb{R}^{m \times n} ; U=\left\{u \in \mathbb{R}^{r}: u_{*} \leqslant u \leqslant u^{*}\right\}-$ множество доступных значений управляющего воздействия, $u_{*}, u^{*} \in \mathbb{R}^{r}$.

Класс доступных управляющих воздействий - дискретные функции (см. [2]) $u(t) \in U, t \in T$, с периодом квантования $h=\left(t^{*}-t_{*}\right) / N(N \in \mathbb{N})$ :

$$
u(t) \equiv u(s), \quad t \in\left[s, s+h\left[, \quad s \in T_{h}=\left\{t_{*}, t_{*}+h, \ldots, t^{*}-h\right\} .\right.\right.
$$

Состояния объекта управления, как правило, отличаются от состояний $x(t), t \in T$, его математической модели (1) в силу неточностей математического моделирования, линеаризации, неизвестных возмущений и других неучтенных неопределенностей. Поэтому состояния объекта будем в дальнейшем обозначать $x^{*}(t), t \in T$. Кроме того, будем считать, что измерения доступны только в дискретные моменты времени $\tau \in T_{h}$.

В настоящем разделе предполагается, что доступны точные и полные измерения (совершенные измерения) состояний, т.е. в каждый момент $\tau \in T_{h}$ для целей управления известно состояние $x^{*}(\tau)$ объекта управления.

В соответствии с предположением о совершенных измерениях, задача оптимального управления (1) погружается в семейство задач

$$
\begin{array}{ll}
c^{\prime} x\left(t^{*}\right) \rightarrow \max , & \\
\dot{x}=A(t) x+B(t) u, & x(\tau)=z, \\
x\left(t^{*}\right) \in X^{*}, & u(t) \in U, \quad t \in T(\tau)=\left[\tau, t^{*}\right],
\end{array}
$$

зависящее от $\tau \in T_{h}$ и $z \in \mathbb{R}^{n}$.

Пусть $u^{0}(t \mid \tau, z), t \in T(\tau)$, - оптимальная программа задачи (2) для позиции $(\tau, z)$ (см. [2]) и $X_{\tau}$ - множество состояний $z$, для которых в момент $\tau \in T_{h}$ оптимальная программа существует.

Функция

$$
u^{0}(\tau, z)=u^{0}(\tau \mid \tau, z), \quad z \in X_{\tau}, \quad \tau \in T_{h},
$$

называется оптимальной (дискретной) обратной связью (позиционным решением задачи (1)).

Пусть оптимальная обратная связь (3) известна. Тогда управление объектом в конкретном процессе осуществляется следующим образом. Процесс начинается в момент $t_{*}$ с подачи на вход объекта управляющего воздействия $u^{*}(t) \equiv u^{*}\left(t_{*}\right):=u^{0}\left(t_{*}, x_{0}^{*}\right), t \geqslant t_{*}$. В момент времени $\tau=t_{*}+h$ измеряется текущее состояние объекта $x^{*}\left(t_{*}+h\right)$. Пусть $\Delta\left(t_{*}+h\right) \in[0, h)$ - время, затрачиваемое на отыскание значения $u^{0}\left(t_{*}+h, x^{*}\left(t_{*}+h\right)\right)$. С момента $t_{*}+h+\Delta\left(t_{*}+h\right)$ на вход объекта подается управляющее воздействие $u^{*}(t) \equiv u^{*}\left(t_{*}+h\right):=u^{0}\left(t_{*}+h, x^{*}\left(t_{*}+h\right)\right), t \geqslant t_{*}+h+\Delta\left(t_{*}+h\right)$. Продолжая этот процесс, в момент времени $\tau \in T_{h}$ измеряется текущее состояние объекта $x^{*}(\tau)$ и с момента $\tau+\Delta(\tau)$ на вход объекта подается управляющее воздействие $u^{*}(t) \equiv u^{*}(\tau):=u^{0}\left(\tau, x^{*}(\tau)\right), t \geqslant$ 
$\tau+\Delta(\tau)$. В результате описанного процесса будет получена последовательность управляющих воздействий

$$
u^{*}(t) \equiv u^{*}(\tau):=u^{0}\left(\tau, x^{*}(\tau)\right), \quad t \in\left[\tau+\Delta(\tau), \tau+h+\Delta(\tau+h)\left[, \quad \tau \in T_{h} .\right.\right.
$$

Функция (4) называется реализацией оптимальной обратной связи в рассматриваемом процессе управления (см. [2,3]).

Нетрудно видеть, что в конкретном процессе управления знать оптимальную обратную связь (3) для всех позиций $(\tau, z), z \in X_{\tau}, \tau \in T_{h}$, не обязательно. Достаточно уметь вычислять значения $u^{*}(\tau)$ ее реализации (4) для реализующихся состояний $x^{*}(\tau)$ по ходу процесса управления, т.е. для каждого $\tau \in T_{h}$.

Будем говорить, что управление объектом осуществляется в реальном времени, если в каждый момент времени $\tau \in T_{h}$ значение $u^{*}(\tau)$ реализации оптимальной обратной связи (4) вычисляется за время $\Delta(\tau)<h$. Соответственно, устройство, способное вычислять реализацию (4) в реальном времени, называется оптимальным регулятором.

Теперь для управления динамическим объектом по совершенным измерениям достаточно реализовать алгоритм работы оптимального регулятора. Алгоритм заключается в следующем.

До начала процесса управления (инициализация алгоритма):

(0) Решить задачу (1), найти ее оптимальную программу $u^{0}\left(t \mid t_{*}, x_{0}\right), t \in T$, и в момент $\tau=t_{*}$ подать на вход объекта управляющее воздействие $u^{*}(t) \equiv u^{*}\left(t_{*}\right)=u^{0}\left(t_{*} \mid t_{*}, x_{0}\right), t \geqslant t_{*}$.

При каждом $\tau \in T_{h} \backslash\left\{t_{*}\right\}$ (по ходу процесса управления, в реальном времени):

(1) Измерить текущее состояние объекта $x^{*}(\tau)$.

(2) Найти оптимальную программу $u^{0}\left(t \mid \tau, x^{*}(\tau)\right), t \in T(\tau)$, задачи (2) при $z=x^{*}(\tau)$.

(3) Подать на вход объекта управляющее воздействие $u^{*}(t) \equiv u^{*}(\tau)=u^{0}\left(\tau \mid \tau, x^{*}(\tau)\right), t \geqslant \tau+\Delta(\tau)$.

Основная трудоемкость приведенного алгоритма работы оптимального регулятора приходится на шаг (2), на котором требуется за время $\Delta(\tau)<h$ решать задачи оптимального управления с сокращающимся промежутком управления $T(\tau)$. Решение задач $(1)$ и $(2)$ при $z=x^{*}(\tau)$ проводится редукцией к задаче линейного программирования (см. [4])

где

$$
\begin{aligned}
& \sum_{\theta \in T_{h}(\tau)} c(\theta)^{\prime} u(\theta) \rightarrow \max , \\
& g_{*} \leqslant \Phi(\tau) x^{*}(\tau)+\sum_{\theta \in T_{h}(\tau)} G(\theta) u(\theta) \leqslant g^{*}, \\
& u_{*} \leqslant u(\theta) \leqslant u^{*}, \quad \theta \in T_{h}(\tau)=T_{h} \cap T(\tau),
\end{aligned}
$$

$$
\begin{gathered}
c(\theta)^{\prime}=\int_{\theta}^{\theta+h} \phi(t)^{\prime} B(t) d t, \quad \phi(t) \in \mathbb{R}^{n}: \dot{\phi}^{\prime}=-\phi^{\prime} A(t), \quad t \in T, \\
\phi\left(t^{*}\right)=c ; \quad G(\theta)=\int_{\theta}^{\theta+h} \Phi(t) B(t) d t, \quad \Phi(t) \in \mathbb{R}^{m \times n}, \\
\dot{\Phi}=-\Phi A(t), \quad t \in T, \quad \Phi\left(t^{*}\right)=H .
\end{gathered}
$$

Для решения задачи (5) рекомендуется использовать специально разработанные в $[4,5]$ алгоритмы, учитывающие исходно динамическую природу задачи линейного программирования (5) и повторяющуюся в каждый текущий момент времени оптимизацию с динамически изменяющимся параметром $x^{*}(\tau)$. В частности, основу предложенный в $[4,5]$ алгоритмов составляют двойственные методы решения задач линейного программирования, в которых выделена структура оптимальной программы (опора и сопровождающие ее элементы), указаны правила пересчета этой структуры на итерациях метода и ее коррекции при переходе от момента времени $\tau$ к моменту $\tau+h$. В [6] предложена модификация метода для линейных стационарных систем, позволяющая ускорить вычисления с помощью рекуррентных вычислений и метода «разновесов». 

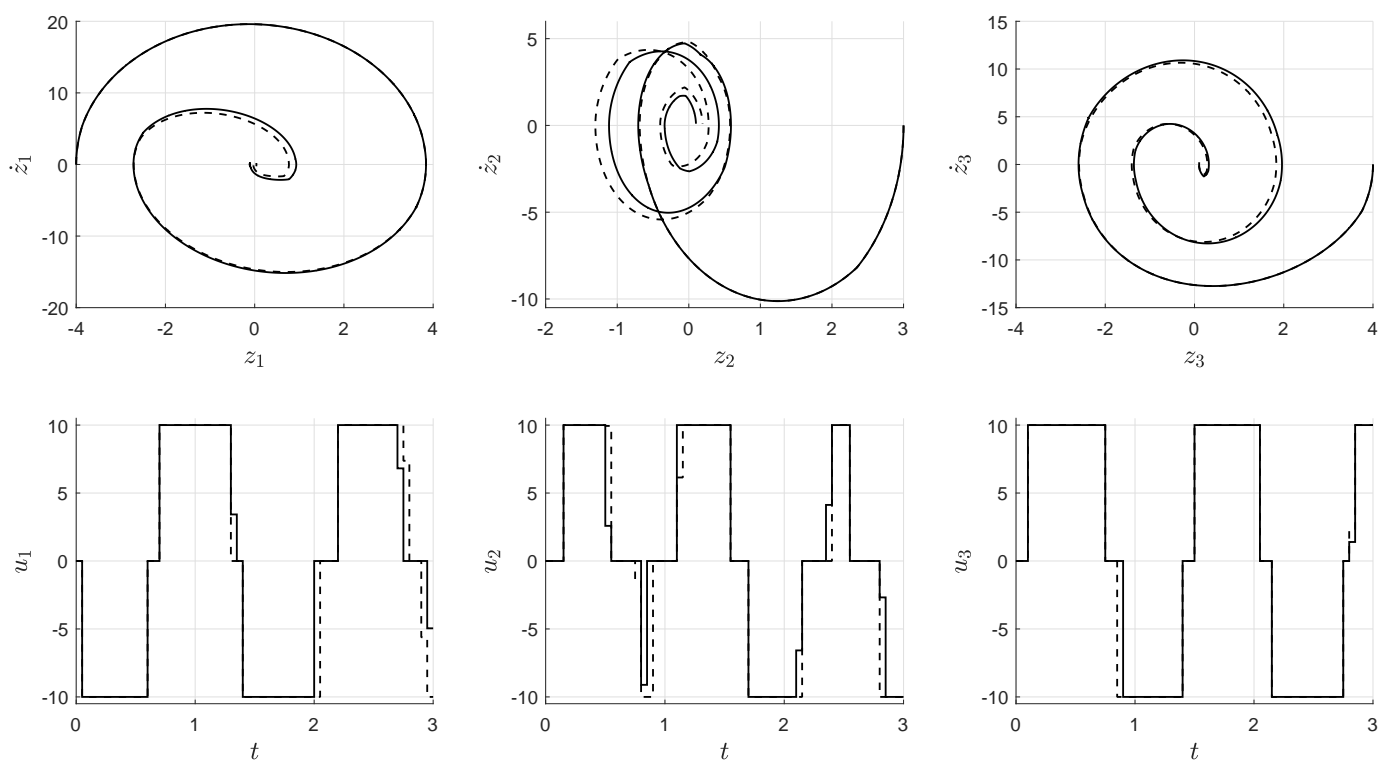

Рис. 1. Сравнение реализации оптимальной обратной связи (сплошная линия) и программного решения (пунктирная линия).

В качестве примера, иллюстрирующего алгоритмы настоящей работы, будем рассматривать задачи оптимального управления для объекта, состоящего из трех взаимосвязанных систем и описываемого линейными уравнениями с аддитивными возмущениями $w$ (см. [15]):

$$
\begin{aligned}
& \ddot{z}_{1}=-2 k z_{1}+k z_{2}+u_{1}+w_{1}, \\
& \ddot{z}_{2}=-2 k z_{2}+k z_{1}+k z_{3}+u_{2}+w_{2}, \\
& \ddot{z}_{3}=-2 k z_{3}+k z_{2}+u_{3}+w_{3} .
\end{aligned}
$$

Пусть управляющие воздействия ограничены по модулю, $\left|u_{i}(t)\right| \leqslant L, t \in\left[0, t^{*}\right], i \in I=\{1,2,3\}$, и в момент времени $t^{*}$ требуется перевести объект (6) на терминальное множество, задаваемое ограничениями $\left|z_{i}\left(t^{*}\right)\right| \leqslant d_{1},\left|\dot{z}_{i}\left(t^{*}\right)\right| \leqslant d_{2}, i \in I$, минимизируя при этом полный импульс управляющего воздействия.

Положим $x_{i}=z_{i}, x_{i+3}=\dot{z}_{i}, i \in I$, и выберем детерминированную модель объекта (6) для построения оптимальной обратной связи; тогда рассматриваемая задача принимает вид

$$
\begin{gathered}
\int_{0}^{t^{*}} \sum_{i \in I}\left|u_{i}(t)\right| d t \rightarrow \min , \\
\dot{x}_{1}=x_{4}, \quad \dot{x}_{4}=-2 k x_{1}+k x_{2}+u_{1}, \\
\dot{x}_{2}=x_{5}, \quad \dot{x}_{5}=-2 k x_{2}+k x_{1}+k x_{3}+u_{2}, \\
\dot{x}_{3}=x_{6}, \quad \dot{x}_{6}=-2 k x_{3}+k x_{2}+u_{3}, \\
x(0)=x_{0}, \quad\left|x_{i}\left(t^{*}\right)\right| \leqslant d_{1}, \quad\left|x_{i+3}\left(t^{*}\right)\right| \leqslant d_{2}, \quad i \in I, \\
\left|u_{i}(t)\right| \leqslant L, \quad t \in\left[0, t^{*}\right], \quad i \in I .
\end{gathered}
$$

Параметрам задачи (7) присвоим следующие значения: $t^{*}=3, k=10, d_{1}=d_{2}=0,1, L=10$.

Для данного численного эксперимента рассматривался процесс, в котором все три системы находились в разных положениях $\left(x_{1}(0)=-4, x_{2}(0)=3, x_{3}(0)=4\right)$ в состоянии покоя $\left(x_{4}(0)=x_{5}(0)=x_{6}(0)=0\right)$. В конкретном процессе управления на объект (6) на промежутке $[0,2]$ действовали постоянные возмущения: $w_{1} \equiv-1, w_{2} \equiv-1, w_{3} \equiv 1$. 
На рис. 1 представлены результаты численного эксперимента: фазовые портреты для трех систем и реализации оптимальной централизованной обратной связи в сравнении с оптимальными программными траекторией и управлением задачи (1). Период квантования равен $h=0,05$.

Оптимальная программа $u^{0}\left(t \mid 0, x_{0}\right), t \in T$, при наличии возмущения не справляется с переводом объекта на терминальное множество: его терминальное состояние оказалось равным $z^{*}\left(t^{*}\right)=(0,0357 ; 0,1924 ; 0,1062), \dot{z}^{*}\left(t^{*}\right)=(-0,2489 ; 0,1081 ; 0,2327)$. При управлении в реальном времени получено $z^{*}\left(t^{*}\right)=(-0,1 ; 0,1 ; 0,1), \dot{z}^{*}\left(t^{*}\right)=(0,1 ; 0,1 ;-0,1)$. Значение критерия качества при оптимальном управлении в реальном времени оказалось равным 64,083666, при оптимальном программном управлении - 67,137245.

3. Оптимальное управление динамическим объектом по недетерминированной модели и несовершенным измерениям. Рассмотрим задачу оптимального управления линейным объектом с недетерминированной моделью и множественной неопределенностью в начальном состоянии:

$$
\begin{array}{ll}
c^{\prime} x\left(t^{*}\right) \rightarrow \max , & \\
\dot{x}=A(t) x+B(t) u+M(t) w(t), & u(t) \in U, t \in T, \\
x\left(t^{*}\right) \in X^{*} & \forall x\left(t_{*}\right) \in X_{0}, \forall w(\cdot) .
\end{array}
$$

В задаче (8) имеют место следующие неопределенности:

(i) возмущение $w(t), t \in T$, неизвестно, но регулярно, т.е. имеет вид $w(t)=L(t) v, t \in T$, где $v \in \mathbb{R}^{l}$ - вектор неизвестных параметров возмущения, $v \in V=\left\{v \in \mathbb{R}^{l}: v_{*} \leqslant v \leqslant v^{*}\right\}-$ априорная неопределенность возмущения;

(ii) начальное состояние $x\left(t_{*}\right)=x_{0}$ объекта управления неизвестно, информация о нем ограничивается включением

$$
x_{0} \in X_{0}=\left\{x \in \mathbb{R}^{n}: d_{*} \leqslant x \leqslant d^{*}\right\},
$$

где $X_{0}$ - априорное распределение начального состояния.

Для уменьшения априорной неопределенности, порожденной множествами $X_{0}$ и $V$, в процессе управления в моменты времени $s \in \bar{T}_{h}=T_{h} \backslash\left\{t_{*}\right\}$ измеряются наблюдаемые входные сигналы $C_{w}(s) w(s)$ и наблюдаемые выходные сигналы $C_{x}(s) x(s)$ с помощью измерительных устройств (cм. [7]):

$$
\begin{array}{ll}
y_{x}(s)=C_{x}(s) x(s)+\xi_{x}(s), & \xi_{x}(s) \in \Xi_{x}, \\
y_{w}(s)=C_{w}(s) w(s)+\xi_{w}(s), & \xi_{w}(s) \in \Xi_{w},
\end{array}
$$

где $C_{x}(t) \in \mathbb{R}^{q_{x} \times n}, C_{w}(t) \in \mathbb{R}^{q_{w} \times n_{w}}, t \in T$, - непрерывные функции, $\xi_{x}(s) \in \mathbb{R}^{q_{x}}, \xi_{w}(s) \in \mathbb{R}^{q_{w}}$, $s \in T_{h}$, - неизвестные ограниченные ошибки измерений,

$$
\Xi_{x}=\left\{\xi_{x} \in \mathbb{R}^{q_{x}}: \xi_{x *} \leqslant \xi_{x} \leqslant \xi_{x}^{*}\right\}, \quad \Xi_{w}=\left\{\xi_{w} \in \mathbb{R}^{q_{w}}: \xi_{w *} \leqslant \xi_{w} \leqslant \xi_{w}^{*}\right\} .
$$

В конкретном процессе управления, в котором к моменту времени $\tau \in T_{h}$ на объект поступило управляющее воздействие $u_{\tau}^{*}=\left(u^{*}(t), t \in\left[t_{*}, \tau[)\right.\right.$, результаты измерений будем обозначать

где $\left.\left.\left.\bar{T}_{h}(\tau)=T_{h} \cap\right] t_{*}, \tau\right]\right)$.

$$
y_{\tau}^{*}=\left\{\left(y_{x}^{*}(s), s \in \bar{T}_{h}(\tau)\right),\left(y_{w}^{*}(s), s \in \bar{T}_{h}(\tau)\right)\right\},
$$

Таким образом, для управления динамическим объектом в каждый момент времени $\tau \in T_{h}$ доступна следующая информация:

(1) априорная неопределенность $\Gamma=X_{0} \times V$;

(2) примененное управляющее воздействие $u_{\tau}^{*}$;

(3) записанные измерения $y_{\tau}^{*}$.

Тройку $\left(\tau, u_{\tau}^{*}, y_{\tau}^{*}\right)$ назовем текущей позицией процесса управления в условиях неопределенности (по недетерминированной модели и несовершенным измерениям).

В соответствии с доступной для управления информацией определим произвольную допустимую позицию процесса управления $\left(\tau, u_{\tau}, y_{\tau}\right)$ для последующего погружения задачи (8) и определения оптимальной обратной связи по измерениям. 
Пару $\left(x_{0}, v\right) \in X_{0} \times V$, назовем согласующейся с управлением $u_{\tau}$ и измерениями $y_{\tau}$, если для соответствующей ей траектории $x\left(t \mid t_{*}, x_{0}, u_{\tau}, v\right), t \in\left[t_{*}, \tau\right]$, системы (8) найдутся такие ошибки измерений $\xi_{x}(s) \in \Xi_{x}, \xi_{w}(s) \in \Xi_{w}, s \in \bar{T}_{h}(\tau)$, что выполняются равенства

$$
\begin{aligned}
& y_{x}(s)=C_{x}(s) x\left(s \mid t_{*}, x_{0}, u_{\tau}, v\right)+\xi_{x}(s), \\
& y_{w}(s)=C_{w}(s) L(s) v+\xi_{w}(s), s \in \bar{T}_{h}(\tau) .
\end{aligned}
$$

Множество $\Gamma\left(\tau, u_{\tau}, y_{\tau}\right)$, состоящее из всех пар $\left(x_{0}, v\right)$, согласующихся с $u_{\tau}, y_{\tau}$, называется апостериорным распределением начального состояния и параметров возмущения (см. [7]).

Задачу оптимального управления (8) погрузим в семейство задач, зависящее от допустимой позиции процесса управления $\left(\tau, u_{\tau}, y_{\tau}\right)$ :

$$
\begin{array}{ll}
\max _{u} \min _{\left(x_{0}, v\right)} c^{\prime} x\left(t^{*}\right), & \\
\dot{x}=A(t) x+B(t) u+M(t) L(t) v, & x(\tau)=x\left(\tau \mid t_{*}, x_{0}, u_{\tau}, v\right), \\
x\left(t^{*}\right) \in X^{*} & \forall\left(x_{0}, v\right) \in \Gamma\left(\tau, u_{\tau}, y_{\tau}\right), u(t) \in U, t \in T(\tau) .
\end{array}
$$

В задаче (10) требуется выполнить терминальные ограничения с гарантией, т.е для всех реализаций начального состояния $x_{0}$ и параметров возмущения $v$, согласующихся с управлением $u_{\tau}$ и измерениями $y_{\tau}$. Кроме того, необходимо максимизировать гарантированное значение терминального критерия качества.

Пусть $u^{0}\left(t \mid \tau, u_{\tau}, y_{\tau}\right), t \in T(\tau)$, - оптимальная гарантирующая программа задачи (10) для позиции $\left(\tau, u_{\tau}, y_{\tau}\right)$ (см. [8]).

Функция

$$
u^{0}\left(\tau, u_{\tau}, y_{\tau}\right)=u^{0}\left(\tau \mid \tau, u_{\tau}, y_{\tau}\right), \quad \tau \in T_{h},
$$

определенная на множестве всех допустимых позиций, для которых существует решение задачи (10), называется оптимальной (дискретной) обратной связью по измерениям.

Тогда реализация оптимальной обратной связи (11) в конкретном процессе управления имеет вид

$$
u^{*}(t) \equiv u^{*}(\tau):=u^{0}\left(\tau, u_{\tau}^{*}, y_{\tau}^{*}\right), \quad t \in\left[\tau+\Delta(\tau), \tau+h+\Delta(\tau+h)\left[, \quad \tau \in T_{h} .\right.\right.
$$

Для построения реализации (12) применим алгоритм работы оптимального регулятора, описанный в разделе 2 , в котором на шаге (2) решается задача (10). Уточним, как эта задача решается в реальном времени для реализованной позиции $\left(\tau, u_{\tau}^{*}, y_{\tau}^{*}\right)$, которой соответствует апостериорное распределение $\Gamma^{*}(\tau)=\Gamma\left(\tau, u_{\tau}^{*}, y_{\tau}^{*}\right)$.

Задача оптимального управления (10) решается редукцией к $2 m+1$ задачам оптимального оценивания состояний системы, содержащей неопределенности

$$
\dot{\hat{x}}=A(t) \hat{x}+M(t) L(t) v, \quad x\left(t_{*}\right)=x_{0}, \quad\left(x_{0}, v\right) \in \Gamma^{*}(\tau),
$$

и одной задаче оптимального управления так называемой номинальной системой

$$
\dot{x}_{0}=A(t) x_{0}+B(t) u(t), \quad x_{0}\left(t_{*}\right)=0, \quad t \in T,
$$

которую требуется перевести на терминальное множество, «суженное» на основе решения задач оптимального оценивания (см. [7]). Такое разбиение на задачи оценивания и управления возможно благодаря хорошо известному свойству разделимости процессов наблюдения и управления в линейных системах.

Пусть $x_{0}^{*}(t), t \in\left[t_{*}, \tau\right],-$ траектория задачи (14) при $u(t)=u^{*}(t), t \in\left[t_{*}, \tau[\right.$. Представим терминальное состояние $x\left(t^{*} \mid t_{*}, x_{0}, u_{\tau}^{*}, v\right)$ в задаче (10) в виде суммы состояния $x_{0}\left(t^{*}\right)$ в момент времени $\tau$ системы (14) с начальным состоянием $x_{0}(\tau)=x_{0}^{*}(\tau)$ и состояния $\hat{x}\left(t^{*} \mid x_{0}, v\right)$ системы (13). Тогда гарантированное значение критерия качества можно вычислить следующим образом:

$$
\min _{\left(x_{0}, v\right) \in \Gamma^{*}(\tau)} c^{\prime} x\left(t^{*}\right)=c^{\prime} x_{0}\left(t^{*}\right)+\min _{\left(x_{0}, v\right) \in \Gamma^{*}(\tau)} c^{\prime} \hat{x}\left(t^{*} \mid x_{0}, v\right),
$$


а выполнение с гарантией $i$-го терминального ограничения эквивалентно выполнению неравенств

$$
\begin{gathered}
g_{* i} \leqslant \min _{\left(x_{0}, v\right) \in \Gamma^{*}(\tau)} h_{i}^{\prime} x\left(t^{*} \mid t_{*}, x_{0}, u_{\tau}^{*}, v\right)=h_{i}^{\prime} x_{0}\left(t^{*}\right)+\min _{\left(x_{0}, v\right) \in \Gamma^{*}(\tau)} h_{i}^{\prime} \hat{x}\left(t^{*} \mid x_{0}, v\right), \\
\max _{\left(x_{0}, v\right) \in \Gamma^{*}(\tau)} h_{i}^{\prime} x\left(t^{*} \mid t_{*}, x_{0}, u_{\tau}^{*}, v\right)=h_{i}^{\prime} x_{0}\left(t^{*}\right)+\max _{\left(x_{0}, v\right) \in \Gamma^{*}(\tau)} h_{i}^{\prime} \hat{x}\left(t^{*} \mid x_{0}, v\right) \leqslant g_{i}^{*},
\end{gathered}
$$

где $h_{i}-i$-я строка матрицы $H, i=\overline{1, m}$.

Отсюда выделяются $2 m+1$ задач оптимального оценивания, состоящих в вычислении оценок множества $\Gamma^{*}(\tau)$ :

$$
\begin{aligned}
& \alpha_{0}(\tau)=\min _{\left(x_{0}, v\right) \in \Gamma^{*}(\tau)} c^{\prime} \hat{x}\left(t^{*} \mid x_{0}, v\right), \\
& \alpha_{i}^{*}(\tau)=\max _{\left(x_{0}, v\right) \in \Gamma^{*}(\tau)} h_{i}^{\prime} \hat{x}\left(t^{*} \mid x_{0}, v\right), \\
& \alpha_{* i}(\tau)=\min _{\left(x_{0}, v\right) \in \Gamma^{*}(\tau)} h_{i}^{\prime} \hat{x}\left(t^{*} \mid x_{0}, v\right), \quad i=\overline{1, m} .
\end{aligned}
$$

Задачи (15) в динамической форме имеют вид

$$
\begin{gathered}
\alpha_{0}(\tau)=\min _{x_{0}, v} c^{\prime} \hat{x}\left(t^{*}\right), \quad \alpha_{i}^{*}(\tau)=\max _{x_{0}, v} h_{i}^{\prime} \hat{x}\left(t^{*}\right), \quad \alpha_{* i}(\tau)=\min _{x_{0}, v} h_{i}^{\prime} \hat{x}\left(t^{*}\right), \\
\dot{\hat{x}}=A(t) \hat{x}+M(t) L(t) v, \quad \hat{x}\left(t_{*}\right)=x_{0}, \\
y_{x}^{*}(s)-C_{x}(s) x_{0}^{*}(s)-C_{x}(s) \hat{x}(s) \in \Xi_{x}, \quad y_{w}^{*}(s)-C_{w}(s) L(s) v \in \Xi_{w}, \quad s \in \bar{T}_{h}(\tau), \\
x_{0} \in X_{0}, \quad v \in V .
\end{gathered}
$$

Задача (10) эквивалентна детерминированной задаче оптимального управления

$$
\begin{array}{ll}
J^{*}(\tau)=\max _{u} c^{\prime} x_{0}\left(t^{*}\right)+\alpha_{0}(\tau), & \\
\dot{x}_{0}=A(t) x_{0}+B(t) u, & x_{0}(\tau)=x_{0}^{*}(\tau), \\
x\left(t^{*}\right) \in X^{\alpha}(\tau), & u(t) \in U, \quad t \in T(\tau),
\end{array}
$$

где

$$
\begin{gathered}
X^{\alpha}(\tau)=\left\{x \in \mathbb{R}^{n}: g_{*}-\alpha_{*}(\tau) \leqslant H x \leqslant g^{*}-\alpha^{*}(\tau)\right\}, \\
\alpha_{*}(\tau)=\left(\alpha_{* i}(\tau), i=\overline{1, m}\right), \quad \alpha^{*}(\tau)=\left(\alpha_{i}^{*}(\tau), i=\overline{1, m}\right) .
\end{gathered}
$$

Уточним алгоритм работы оптимального регулятора, реализующего оптимальную обратную связь по измерениям.

До начала процесса управления (инициализация алгоритма):

(0) решить $2 m+1$ задач (15) при условиях $\left(x_{0}, v\right) \in X_{0} \times V$, найти $\alpha_{0}\left(t_{*}\right), \alpha_{*}\left(t_{*}\right), \alpha^{*}\left(t_{*}\right)$. Решить задачу (17) с $x_{0}\left(t_{*}\right)=0$, найти ее оптимальную программу $u^{0}(t), t \in T$, и в момент $\tau=t_{*}$ подать на вход объекта управляющее воздействие $u^{*}(t) \equiv u^{*}\left(t_{*}\right)=u^{0}\left(t_{*}\right), t \geqslant t_{*}$.

При каждом $\tau \in T_{h} \backslash\left\{t_{*}\right\}$ (по ходу процесса управления, в реальном времени):

(1) получить измерения $y_{x}^{*}(\tau), y_{w}^{*}(\tau)$;

(2) решить $2 m+1$ задач $(16)$, найти $\alpha_{0}(\tau), \alpha_{*}(\tau), \alpha^{*}(\tau)$;

(3) найти оптимальную программу $u^{0}\left(t \mid \tau, u_{\tau}^{*}, y_{\tau}^{*}\right), t \in T(\tau)$, задачи (17);

(4) подать на вход объекта управляющее воздействие $u^{*}(t) \equiv u^{*}(\tau)=u^{0}\left(\tau \mid \tau, u_{\tau}^{*}, y_{\tau}^{*}\right), t \geqslant \tau+\Delta(\tau)$.

Методы решения задач оптимального оценивания, учитывающие их особенности, в частности, повторяющуюся в каждый момент $\tau \in T_{h}$ оптимизацию, разработаны в [6,8].

Для приведенного алгоритма работы оптимального регулятора имеет место следующее утверждение.

Теорема 1. Пусть на шаге (0) алгоритма задача оптимального управления имеет решение. Тогда при любом $\tau \in T_{h} \backslash\left\{t_{*}\right\}$

(i) задачи (16), (17), имеют решение;

(ii) оптимальное значение задачи (17) в процессе управления не ухудиается: $J^{*}(\tau-h) \leqslant J^{*}(\tau)$. 

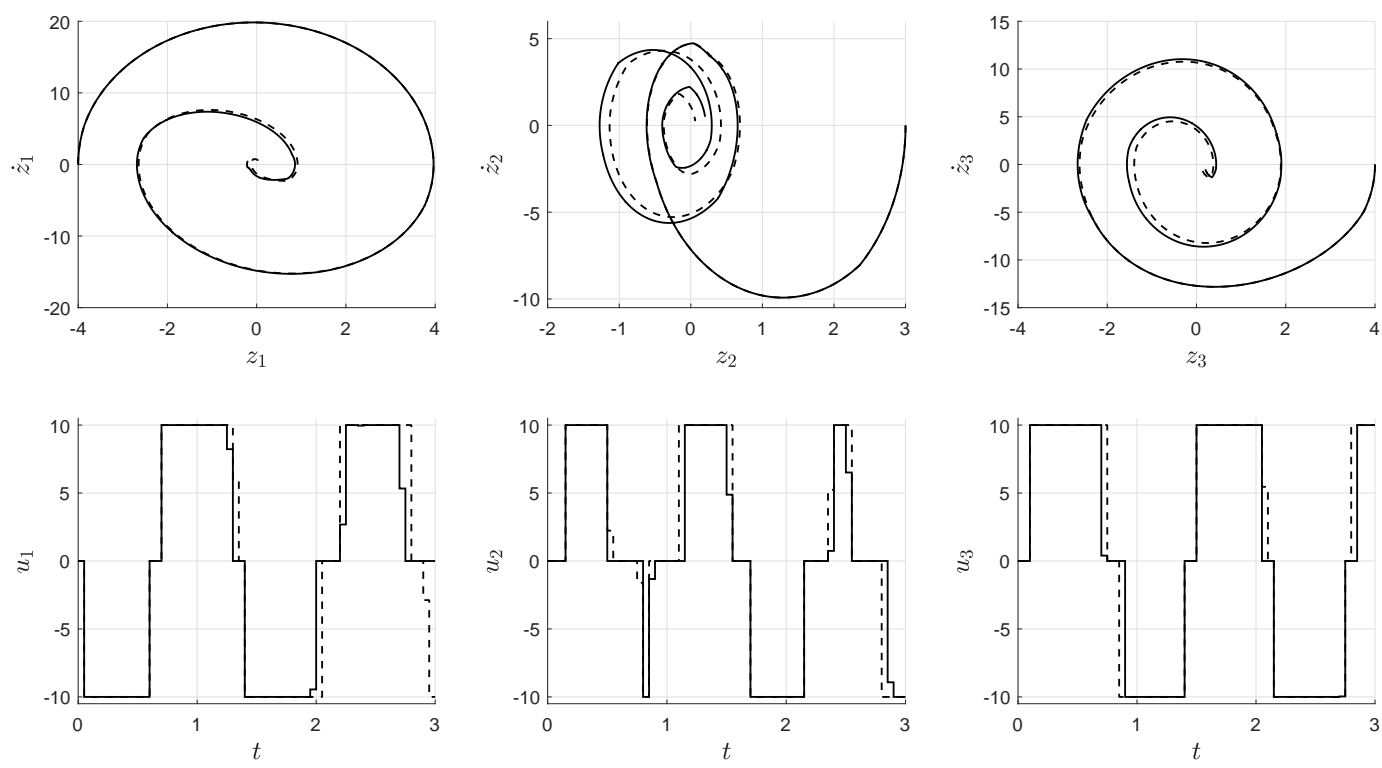

Рис. 2. Сравнение реализации оптимальной обратной связи (сплошная линия) и программного решения (пунктирная линия).

Отметим, что для алгоритма раздела 2 невозможно гарантировать выполнение ни одного из условий теоремы 1 , поскольку управление объектом осуществляется по его детерминированной модели, неопределенности игнорируются.

Для иллюстрации результатов данного раздела рассмотрим пример раздела 2 , принимая уравнения (6) в качестве математической модели с возмущениями.

Пусть априорное распределение начального состояния задается условиями

$$
x_{1}(0)=-4, \quad x_{2}(0)=3, \quad x_{3}(0)=4, \quad\left|x_{i+3}(0)\right| \leqslant \epsilon,
$$

а регулярные возмущения имеют вид

$$
\begin{gathered}
w_{1}(t)=-v_{1}, \quad w_{2}(t)=v_{2} \sin t, \quad w_{3}(t)=v_{3} \cos 3 t, \quad t \in T, \\
v \in V=\left\{v \in \mathbb{R}^{3}:\left\|v_{i}\right\|_{\infty} \leqslant v_{\max }\right\} .
\end{gathered}
$$

Измерительные устройства (9) измеряют с ошибками положение систем и суммарное возмущение равно

$$
\begin{aligned}
& y_{x i}(s)=z_{i}(s)+\xi_{x i}(s), \quad\left|\xi_{x i}(s)\right| \leqslant \epsilon_{x}, \quad i \in I, \\
& y_{w}(s)=\sum_{i \in I} w_{i}(s)+\xi_{w}(s), \quad\left|\xi_{w}(s)\right| \leqslant \epsilon_{w}, \quad s \in \bar{T}_{h} .
\end{aligned}
$$

Параметрам задачи присвоим следующие значения:

$$
\epsilon=\epsilon_{x}=0,2, \quad \epsilon_{w}=0,1, \quad v_{\max }=0,5, \quad d_{1}=0,2, \quad d_{2}=0,5 .
$$

На рис. 2 представлены результаты численного эксперимента для процесса, в котором реализовалось начальное состояние как в примере раздела 2 , вектор $v=(0,1 ;-0,4 ; 0,3)$ и некоторые случайные ошибки измерений $\xi_{x i}, \xi_{w}$. Период квантования равен $h=0,05$.

На рис. 3 показано, как изменяются оценки неопределенности - решения задач (16). Сближение верхних $\alpha_{i}^{*}$ и нижних $\alpha_{* i}$ оценок означает уменьшение неопределенности по ходу процесса управления.

Значение критерия качества на оптимальной программе оказалось равным $J^{*}(0)=67,666113$, тогда как реализация оптимальной обратной связи позволяется добиться существенного улучшения качества процесса управления: $J^{*}\left(t^{*}\right)=61,419896$. 

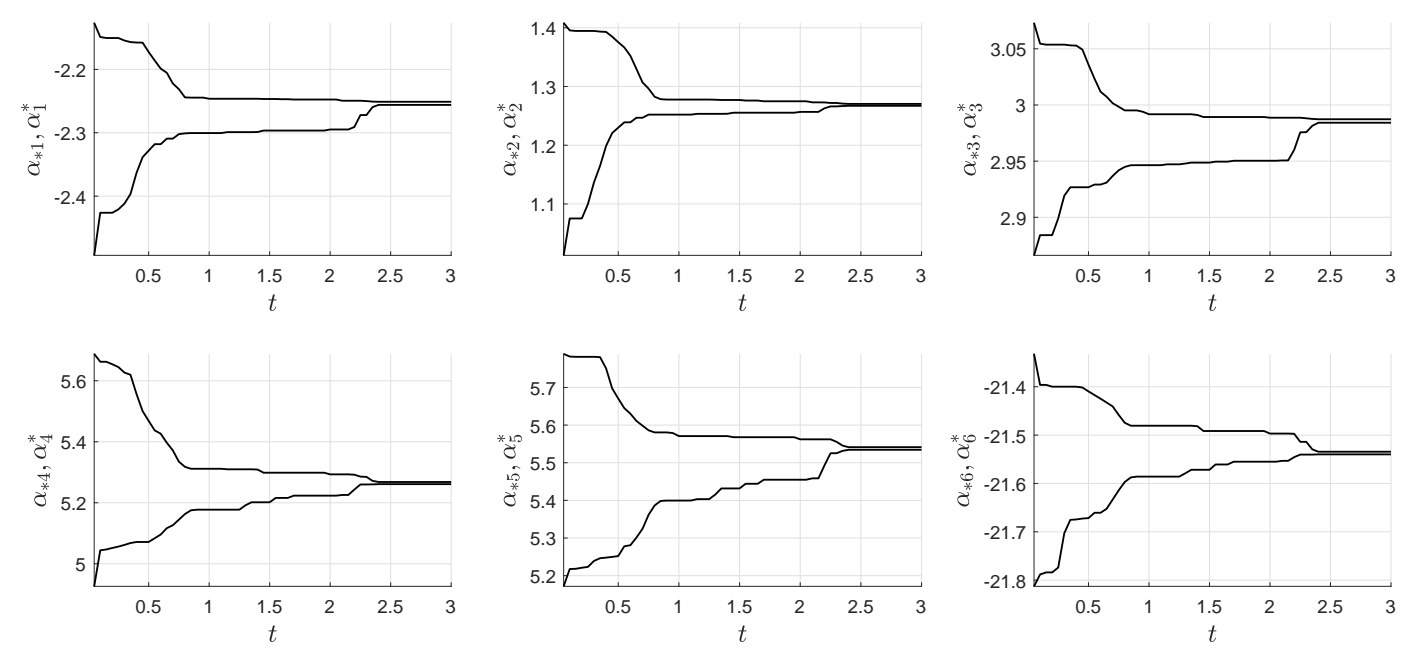

Рис. 3. Изменение оценок $\alpha_{* i}, \alpha_{i}^{*}, i=\overline{1,6}$.

4. Децентрализованное управление в реальном времени. Принцип управления в реальном времени, описанный в разделах 2,3 , допускает естественное развитие на задачи оптимального управления группами динамических систем (см. $[9,10,12,13,15])$, в которых каждый объект управляется децентрализованно, т.е. имеет свой собственный локальный оптимальный регулятор.

На промежутке времени $T$ рассмотрим группу, состоящую их $q$ линейных динамических объектов, в которой поведение $i$-го объекта, $i \in I=\{1,2, \ldots, q\}$, описывается уравнением с возмущением

$$
\dot{x}_{i}=A_{i}(t) x_{i}+\sum_{j \in I_{i}} A_{i j}(t) x_{j}+B_{i}(t) u_{i}+M_{i}(t) w_{i}, \quad x_{i}(0)=x_{i 0} .
$$

Здесь $x_{i}=x_{i}(t) \in \mathbb{R}^{n_{i}}, u_{i}=u_{i}(t) \in U_{i} \subset \mathbb{R}^{r_{i}}, w_{i}=w_{i}(t) \in W_{i} \subset \mathbb{R}^{p_{i}}$ - состояние, управление и возмущение $i$-го объекта в момент времени $t ; U_{i}$ и $W_{i}$ - выпуклые компакты, содержащие начало координат. Кусочно непрерывные матричные функции $A_{i}(t) \in \mathbb{R}^{n_{i} \times n_{i}}, B_{i}(t) \in \mathbb{R}^{n_{i} \times r_{i}}, M_{i}(t) \in$ $\mathbb{R}^{n_{i} \times p_{i}}, t \in T$, описывают собственную динамику, входное устройство и влияние возмущений $i$ го объекта группы, $A_{i j}(t), j \in I_{i}=I \backslash i, t \in T$, описывают динамические взаимосвязи между объектами.

Как и в разделе 2, для управления каждым объектом (18) используются дискретные управляющие воздействия $u_{i}(t), t \in T$. Кроме того, предполагается, что в моменты $\tau \in T_{h}$ проводятся совершенные измерения $x_{i}^{*}(\tau)$ состояний каждого объекта $i \in I$, однако в зависимости от схемы управления (см. ниже) они могут быть доступны не всем объектам.

Кроме динамической взаимосвязи между объектами (18), в терминальный момент времени $t^{*}$ зададим статические связи на состояния в следующем виде:

$$
g_{*} \leqslant \sum_{k \in I} H_{k} x_{k}\left(t^{*}\right) \leqslant g^{*}
$$

где $H_{k} \in \mathbb{R}^{m \times n_{k}}, k \in I, g_{*}, g^{*} \in \mathbb{R}^{m}$. Поскольку (18) содержат возмущения, ограничения (19) требуется выполнить с гарантией, т.е. при всех возможных возмущениях (см. [9]).

Целью управления группой объектов является максимизация гарантированного значения общего терминального критерия качества

$$
\min _{w_{k}, k \in I} \sum_{k \in I} c_{k}^{\prime} x_{k}\left(t^{*}\right) \rightarrow \max .
$$


При оптимальном централизованном управлении в реальном времени группа (18), $i \in I$, трактуется как одна большая динамическая система

$$
\dot{x}=A(t) x+B(t) u+M(t) w, \quad x(0)=x_{0},
$$

где $x=\left(x_{1}^{\prime}, \ldots, x_{q}^{\prime}\right)^{\prime}, u=\left(u_{1}^{\prime}, \ldots, u_{q}^{\prime}\right)^{\prime}, w=\left(w_{1}^{\prime}, \ldots, w_{q}^{\prime}\right)^{\prime} ; A(t), B(t), M(t)$ - соответствующие блочные матрицы.

Система (20) имеет единый центр управления - центральный оптимальный регулятор, которому доступны для целей управления измерения всех текущих состояний объектов $x^{*}(\tau)=$ $\left(x_{1}^{*}(\tau)^{\prime}, \ldots x_{q}^{*}(\tau)^{\prime}\right)^{\prime}$, и который вырабатывает реализацию (централизованной) оптимальной обратной связи вида (4) для всех объектов группы.

Алгоритм работы центрального оптимального регулятора повторяет описанный в разделе 2 , с той лишь разницей, что решаемая на шаге (2) задача оптимального управления формулируется для номинальной системы $(w \equiv 0)$, соответствующей $(20)$, и имеет вид (аналогично разделу 3$)$

$$
\begin{array}{ll}
\max _{u} c^{\prime} x_{0}\left(t^{*}\right)+\gamma_{0}(\tau), & \\
\dot{x}_{0}=A(t) x_{0}+B(t) u, & x(\tau)=x^{*}(\tau), \\
x_{0}\left(t^{*}\right) \in X^{\gamma}(\tau), & u(t) \in U, \quad t \in T(\tau),
\end{array}
$$

где $c=\left(c_{1}^{\prime}, \ldots, c_{q}^{\prime}\right)^{\prime}$,

$$
\begin{gathered}
X^{\gamma}(\tau)=\left\{x \in \mathbb{R}^{n}: g_{*}-\gamma_{*}(\tau) \leqslant H x \leqslant g^{*}-\gamma^{*}(\tau)\right\}, \\
H=\left(H_{1}, \ldots, H_{q}\right), \quad U=U_{1} \times \ldots \times U_{q}, \quad W=W_{1} \times \ldots \times W_{q} .
\end{gathered}
$$

Величины $\gamma_{0}(\tau), \gamma_{*}(\tau), \gamma^{*}(\tau)$ - оценки наихудших реализаций возмущений по отношению к критерию качества и терминальным ограничениям задачи оптимального управления. Они вычисляются по правилам

$$
\gamma_{0}(\tau)=\int_{\tau}^{t^{*}} \min _{w \in W} \phi(t)^{\prime} M(t) w d t, \quad \gamma_{*}(\tau)=\int_{\tau}^{t^{*}} \min _{w \in W} \Phi(t) M(t) w d t, \quad \gamma^{*}(\tau)=\int_{\tau}^{t^{*}} \max _{w \in W} \Phi(t) M(t) w d t,
$$

где операции минимума и максимума применяются к векторной функции покомпонентно.

При оптимальном децентрализованном управлении каждый $i$-й объект имеет собственный (локальный) регулятор, которому доступны для целей управления измерение состояния собственного объекта $x_{i}^{*}(\tau)$ и некоторая информация о поведении других объектов (см. $\left.[9,10,12,13]\right)$.

В системах децентрализованного управления, как правило, предполагается что в канале обмена информацией между локальными регуляторами имеется запаздывание, равное периоду квантования $h$ (см. $[13,15])$. Информация, поступающая от локального регулятора $k$-го объекта, обозначается $z_{k}^{*}(\tau-h)$ и обсуждается в конце настоящего раздела.

Локальный оптимальный регулятор в режиме реального времени вырабатывает только локальное управляющее воздействие $u_{i}^{*}(t), t \in T_{h}$ для своего объекта.

Инициализация алгоритма децентрализованного управления проводится с помощью центрального регулятора, который решает задачу (21). Начиная с момента $\tau=h$, центральный регулятор не используется.

Для нахождения в текущий момент времени $\tau \in T_{h} \backslash\left\{t_{*}\right\}$ управляющего воздействия $u_{i}^{*}(t)$, $t \in[\tau, \tau+h[$, регулятор $i$-го объекта решает локальную задачу оптимального управления, которую будем обозначать $P_{i}\left(\tau, x_{i}^{*}(\tau), z_{k}^{*}(\tau-h), k \in I_{i}\right)$ или, для сокращения записи, $P_{i}(\tau)$.

Если $u_{i}^{d}(t \mid \tau), t \in T(\tau)$, - решение задачи $P_{i}(\tau)$, то, начиная с момента времени $\tau+\Delta_{i}(\tau)$, где $\Delta_{i}(\tau)$ - время решения задачи $P_{i}(\tau)$, на вход $i$-го объекта группы подается управляющее воздействие

$$
u_{i}^{*}(t)=u_{i}^{d}(\tau \mid \tau), \quad t \in\left[\tau+\Delta_{i}(\tau), \tau+h+\Delta_{i}(\tau+h)\left[, \quad \tau \in T_{h} .\right.\right.
$$

Функция (22), $i \in I$, называется реализацией оптимальной (децентрализованной) обратной связи в конкретном процессе управления.

Суммируя, алгоритм оптимального децентрализованного управления состоит в следующем. 
До начала процесса управления (инициализация алгоритма):

(0) найти решение $u^{0}\left(t \mid t_{*}, x_{0}\right), t \in T$, задачи оптимального управления (21) при $\tau=t_{*}, x^{*}(\tau)=x_{0}$. Подать на объекты группы управляющие воздействия $u_{i}^{*}(t) \equiv u_{i}^{*}\left(t_{*}\right):=u_{i}^{0}\left(t_{*} \mid t_{*}, x_{0}\right), t \geqslant t_{*}$, $i \in I$. Вычислить и передать всем локальным регуляторам $z_{i}^{*}\left(t_{*}\right), i \in I$.

При каждом $\tau \in T_{h} \backslash\left\{t_{*}\right\}$ для каждой подсистемы $i \in I$ параллельно (в реальном времени):

(1) решить задачу $P_{i}(\tau)$ и найти $u_{i}^{d}(t \mid \tau), t \in T(\tau)$;

(2) подать на вход $i$-го объекта управляющее воздействие $u_{i}^{*}(t) \equiv u_{i}^{*}(\tau):=u_{i}^{d}(\tau \mid \tau), t \geqslant \tau+\Delta_{i}(\tau)$;

(3) передать локальную информацию $z_{i}^{*}(\tau)$ всем остальным регуляторам $k \in I_{i}$.

Следуя идеям работ [9,12], локальную (детерминированную) задачу оптимального управления $P_{i}(\tau)$ для $i$-го регулятора в момент времени $\tau \in T_{h} \backslash\left\{t_{*}\right\}$ сформируем следующим образом:

$$
\begin{array}{ll}
J_{i}^{d}(\tau)=\max _{u_{i}} \sum_{k \in I} c_{k}^{\prime} x_{k}\left(t^{*}\right), & \\
\dot{x}_{i}=A_{i}(t) x_{i}+\sum_{j \in I_{i}} A_{i j}(t) x_{j}+B_{i}(t) u_{i}, & x_{i}(\tau)=x_{i}^{*}(\tau), \\
\dot{x}_{k}=A_{k}(t) x_{k}+\sum_{j \in I_{k}} A_{k j}(t) x_{j}, & x_{k}(\tau)=0, \quad k \in I_{i}, \\
x\left(t^{*}\right) \in X_{i}^{\gamma}(\tau), & u_{i}(t) \in U_{i}, \quad t \in T(\tau),
\end{array}
$$

где

$$
\begin{gathered}
X_{i}^{\gamma}(\tau)=\left\{x: g_{* i}(\tau)-\gamma_{* i}(\tau) \leqslant \sum_{k \in I} H_{k} x_{k} \leqslant g_{i}^{*}(\tau)-\gamma_{i}^{*}(\tau)\right\}, \\
g_{* i}(\tau)=y_{i}^{d}(\tau)+\Omega_{* i}(\tau)\left[g_{*}-\sum_{k \in I} y_{k}^{*}(\tau-h)\right] \\
g_{i}^{*}(\tau)=y_{i}^{d}(\tau)+\Omega_{i}^{*}(\tau)\left[g^{*}-\sum_{k \in I} y_{k}^{*}(\tau-h)\right], \\
y_{i}^{d}(\tau)=\Phi_{i}(\tau) x_{i}^{d}(\tau \mid \tau-h)+\int_{\tau}^{t^{*}} \Phi_{i}(t) B_{i}(t) u_{i}^{d}(t \mid \tau-h) d t, \\
y_{k}^{*}(\tau)=\Phi_{k}(\tau) x_{k}^{*}(\tau)+\int_{\tau}^{t^{*}} \Phi_{k}(t) B_{k}(t) u_{k}^{d}(t \mid \tau) d t, \\
\gamma_{* i}(\tau)=\int_{\tau}^{t^{*}} \min _{w_{i} \in W_{i}} \Phi_{i}(t) M_{i}(t) w_{i} d t, \quad \gamma_{i}^{*}(\tau)=\int_{\tau} \max _{w_{i} \in W_{i}} \Phi_{i}(t) M_{i}(t) w_{i} d t,
\end{gathered}
$$

$x^{d}(\tau \mid \tau-h)=\left(x_{1}^{d}(\tau \mid \tau-h)^{\prime}, \ldots, x_{q}^{d}(\tau \mid \tau-h)^{\prime}\right)^{\prime}$ - состояние в момент $\tau$ номинальной системы $(21)$ с начальным условием $x_{0}(\tau-h)=x^{*}(\tau-h)$ и управлением $u(t) \equiv u^{*}(\tau-h), t \in[\tau-h, \tau]$; $\left(\Phi_{1}(t), \ldots, \Phi_{q}(t)\right)=\Phi(t) ; \Omega_{* i}, \Omega_{i}^{*} \in \mathbb{R}^{m \times m}$ - весовые коэффициенты,

$$
\sum_{i \in I} \Omega_{* i}=\sum_{i \in I} \Omega_{i}^{*}=E .
$$

Из формулировки задачи (23) следует, что для ее построения локальному регулятору объекта $i$ достаточно получить к моменту $\tau$ от остальных регуляторов следующую информацию о поведении их объектов: $z_{k}^{*}(\tau-h)=\left\{x_{k}^{*}(\tau-h), u_{k}^{*}(\tau-h), y_{k}^{*}(\tau-h)\right\}$. Именно эта информация передается на шаге (3) алгоритма.

Отметим, что при реализации приведенного алгоритма децентрализованного управления возможна ситуация, когда в некоторый момент $\tau$ одна или несколько локальных задач оптимального 
управления (23) не имеет решения. Обычно это связано с возмущениями, долгое время находящимися на границе множеств их возможных значений. Для того, чтобы избежать подобных ситуаций, в [12] предлагается заменить оценки $\gamma$ в задачах $(21),(23)$ на величины $\delta$ и строить терминальные множества $X^{\delta}(\tau), X_{i}^{\delta}(\tau)$, где

$$
\begin{gathered}
\delta_{*}(\tau)=\sum_{k \in I} \delta_{* i}(\tau), \quad \delta^{*}(\tau)=\sum_{k \in I} \delta_{i}^{*}(\tau), \\
\delta_{* i}(\tau)=\sum_{s \in T_{h}(\tau)} \min _{w} \Phi_{i}(s+h) x_{i}(s+h \mid s, w), \\
\delta_{i}^{*}(\tau)=\sum_{s \in T_{h}(\tau)} \max _{w} \Phi_{i}(s+h) x_{i}(s+h \mid s, w),
\end{gathered}
$$

$x(s+h \mid s, w)$ - состояние в момент $s+h$ системы (18) с начальным условием $x(s)=0$, управлением $u(t) \equiv 0, t \in[s, s+h]$, и возмущением $w(t), t \in[s, s+h]$.

Тогда можно доказать следующий результат.

Теорема 2. Пусть в момент $\tau=t_{*}$ задача (21) с терминальным множеством $X^{\delta}\left(t_{*}\right)$ имеет решение, а веса $\Omega_{* i}(\tau), \Omega_{i}^{*}, i \in I$, таковы, что

$$
\delta_{* i}(\tau-h)=\Omega_{* i}(\tau) \delta_{*}(\tau-h), \quad \delta_{i}^{*}(\tau-h)=\Omega_{i}^{*}(\tau) \delta^{*}(\tau-h), \quad i \in I, \tau \in T_{h} \backslash\left\{t_{*}\right\} .
$$

Тогда при любом $\tau \in T_{h} \backslash\left\{t_{*}\right\}$ справедливы следующие утверждения:

(i) все локальные задачи $P_{i}(\tau), i \in I$, с терминальными множествами $X_{i}^{\delta}(\tau)$ разрешимы;

(ii) агрегированная децентрализованная программа

$$
u^{d}(t \mid \tau)=\left(u_{k}^{d}(t \mid \tau), k \in I\right), \quad t \in T(\tau),
$$

является программой задачи (21), при этом значение критерия качества равно

$$
J^{d}(\tau)=\gamma_{0}(\tau)+\sum_{k \in I} J_{k}^{d}(\tau)
$$

и отклоняется от оптимального на величину $O\left(\tau w_{\max }\right)$, где $w_{\max }=\max _{w \in W}\|w\|_{\infty}$;

(iii) значение критерия качества в прочессе управления не ухудшается:

$$
J^{d}(\tau-h) \leqslant J^{d}(\tau) .
$$

Для иллюстрации работы алгоритма оптимального децентрализованного управления и сравнения качества реализаций оптимальной централизованной и децентрализованной обратной связи рассмотрим пример (7) раздела 2 с математической моделью $(6)$, где $\left|w_{i}\right| \leqslant w_{\max }, i \in I$. Положим $w_{\max }=0.5$ и изменим параметры задачи, касающиеся терминального множества: $d_{1}=0,5, d_{2}=2$.

На рис. 4 представлены результаты численного эксперимента: фазовые портреты для трех объектов и реализации оптимальной централизованной (сплошная линия) и децентрализованной (пунктирная линия) обратной связи. Период квантования равен $h=0,05$. Значение критерия качества при централизованном управлении оказалось равным 50,866405, при децентрализованном - 52,147609. Потеря качества составила $2,51 \%$.

5. Задачи стабилизации линейных динамических объектов. Задачи оптимального управления находят применение при решении различных проблем теории управления, в своей постановке не являющихся оптимизационными. Примером таких задач могут служить задачи стабилизации линейных систем

$$
\dot{x}=A x+B u, \quad x(0)=x_{0},
$$

ограниченными стабилизирующими обратными связями, т.е. при выполнении дополнительного требования к применяемым к системе управляющим воздействиям вида

$$
u(t) \in U=\left\{u \in \mathbb{R}^{r}:\|u\|_{\infty} \leqslant L\right\} .
$$



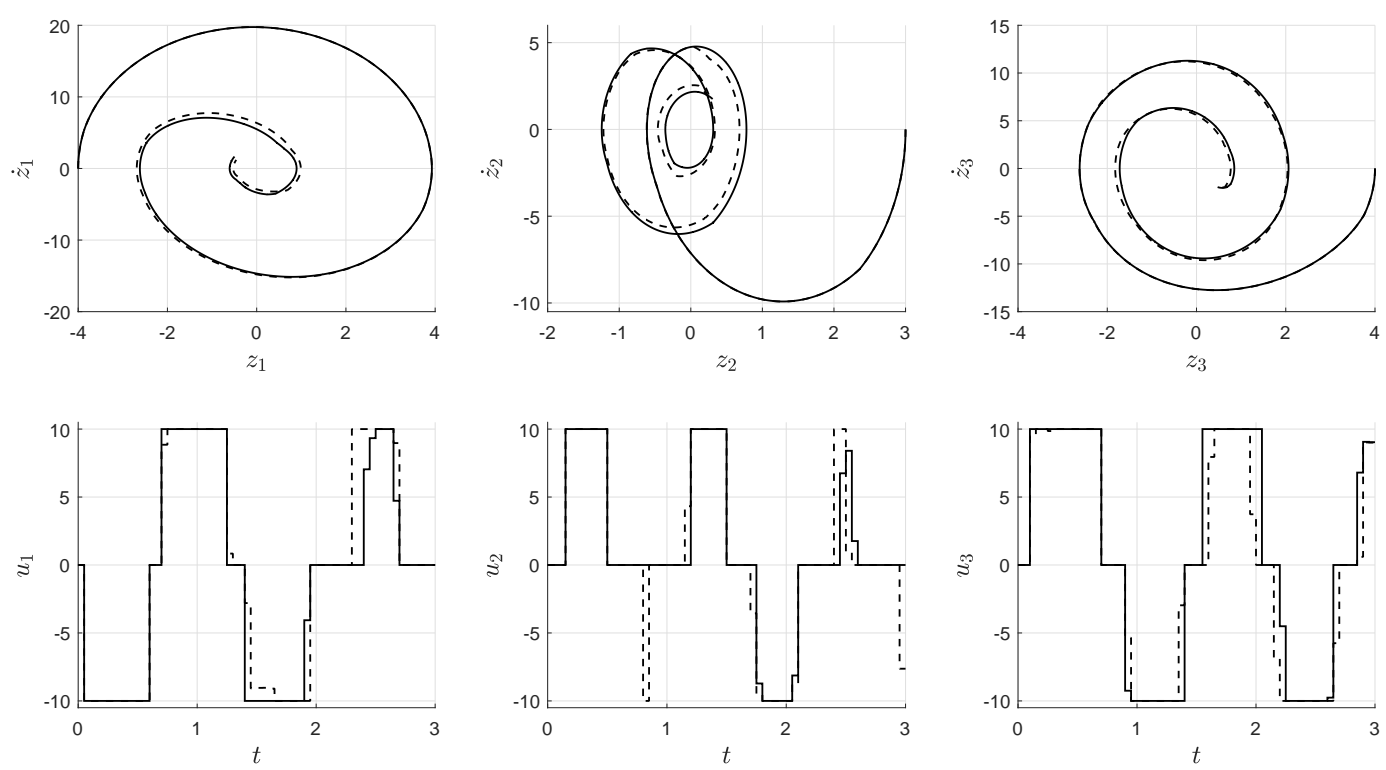

Рис. 4. Сравнение оптимального централизованного (сплошная линия) и децентрализованного (пунктирная линия) управления

Для определения ограниченной стабилизирующей обратной связи предлагается использовать задачу оптимального управления с конечным промежутком $T=\left[0, t^{*}\right]$, и параметром $z \in \mathbb{R}^{n}$ (см. [1]):

$$
\rho(z)=\min _{u} \rho, \quad \dot{x}=A x+B u, \quad x(0)=z, x\left(t_{f}\right)=0, \quad\|u(t)\|_{\infty} \leqslant \rho, t \in T .
$$

При предположениях об управляемости системы (24) и выполнении требования $t^{*}>h n / r$ каждая задача семейства (25), $z \in \mathbb{R}^{n}$, имеет решение, которое обозначается $u^{0}(t \mid z), t \in T$.

Пусть $D=\left\{z \in \mathbb{R}^{n}: \rho(z) \leqslant L\right\}$. Для всех $z \in D$ выполняется включение $u^{0}(t \mid z) \in U, t \in T$, по построению.

В [1] получен следующий результат.

Теорема 3. Функиия $u^{0}(x)=u^{0}(0 \mid x), x \in D$, является ограниченной стабилизирующей обратной связъю для замкнутой системъь

$$
\dot{x}=A(t) x+B(t) u(x(t)), \quad x(0)=x_{0},
$$

т.е. справедливы следующие утверждения:

(i) $u(x) \in U, x \in D ; u(0)=0$;

(ii) для любого $x_{0} \in D$ состояния $x(s), s \in T_{h}^{\infty}=\{0, h, 2 h, \ldots\}$, системы (26) остаятся в $D$;

(iii) нулевое решение $x(t) \equiv 0, t \geqslant 0$, системы (26) асимптотически устойчиво в $D$.

Алгоритм стабилизации системы (24) состоит в следующем.

До начала процесса управления (инициализация алгоритма):

(0) решить задачу (25) при $\tau=0, z=x_{0}$, найти ее оптимальную программу $u^{0}\left(t \mid x_{0}\right), t \in T$, и в момент $\tau=0$ подать на вход объекта управляющее воздействие $u^{*}(t) \equiv u^{0}\left(x_{0}\right):=u^{0}\left(0 \mid x_{0}\right)$, $t \geqslant 0$.

При каждом $\tau \in T_{h}^{\infty} \backslash\{0\}$ (в реальном времени):

(1) измерить текущее состояние объекта $x^{*}(\tau)$;

(2) найти оптимальную программу $u^{0}\left(t \mid x^{*}(\tau)\right), t \in T(\tau)$, задачи (25) при $z=x^{*}(\tau)$; 

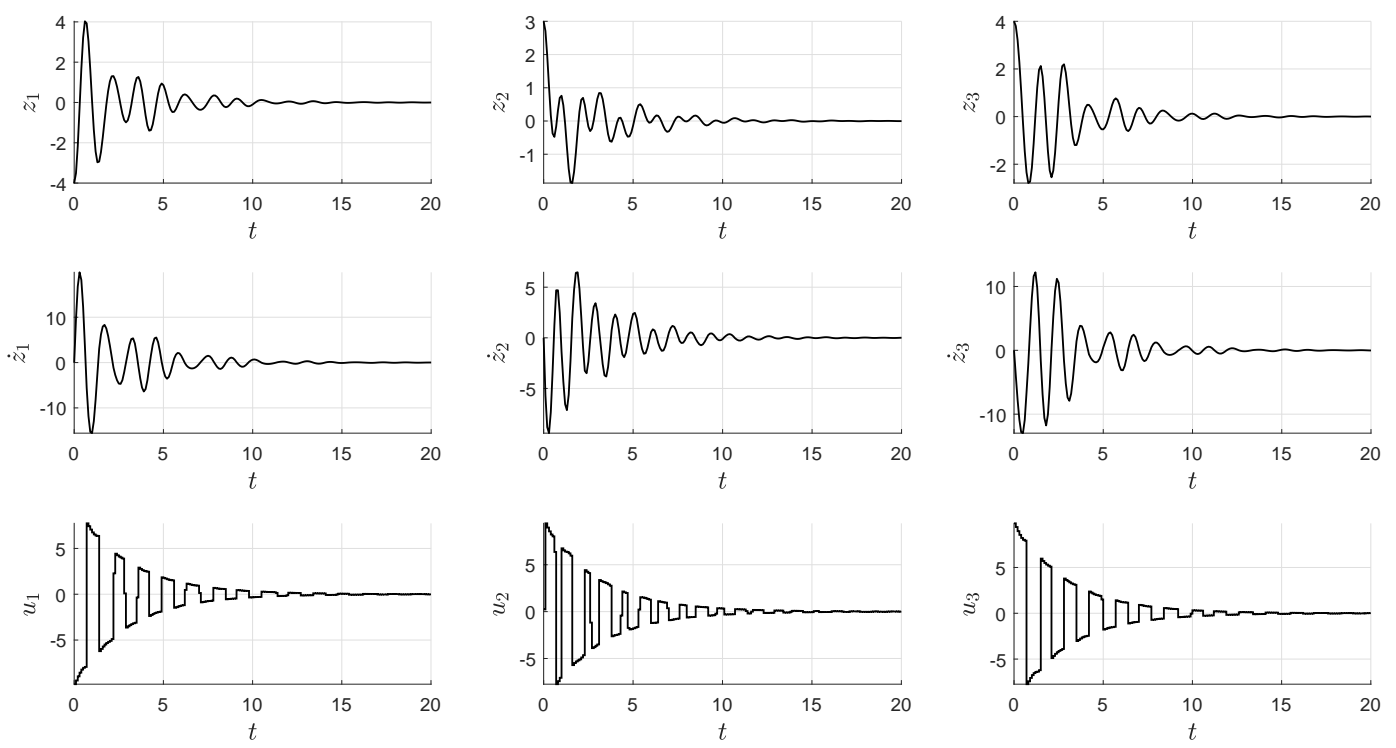

Рис. 5. Фазовые траектории замкнутой системы и реализация стабилизирующей обратной связи

(3) gодать на вход динамического объекта управляющее воздействие

$$
u^{*}(t)=u^{0}\left(x^{*}(\tau)\right)=u^{0}\left(0 \mid x^{*}(\tau)\right), \quad t \geqslant \tau+\Delta(\tau) .
$$

Для решения задачи (25) на шаге (2) приведенного алгоритма также могут использоваться эффективные методы, предложенные в [4-6].

Для иллюстрации результата снова рассмотрим объект, состоящий из трех систем (6) при тех же начальных условиях, что в разделе 2 , и ограничении на управляющие воздействия $L=10$. Горизонт управления в задачеЁ $(25)$ выбран $t^{*}=3$.

На рис. 5 представлены результаты численного эксперимента для $\tau \in\{0, h, \ldots, 200 h\}, h=$ 0,1. Выбранное начальное состояние принадлежит области $D$, поскольку $\rho\left(x_{0}\right)=9,8175<L$. В процессе стабилизации к моменту $\tau=40,9$ получены значения $\rho(\tau)<10^{-5},\left\|x^{*}(\tau)\right\|<3 \times$ $10^{-5}$, полный импульс поданных на вход объекта управляющих воздействий к этому моменту составил 84,3109.

Отметим, что методы решения различных проблем теории управления линейных и нелинейных систем на основе решения в реальном времени задач оптимального управления на конечном промежутке развиваются в литературе в рамках теории управления по прогнозирующей модели (см. [16]). Основное отличие представленного в настоящем разделе результата состоит в выборе критерия качества задачи (25), более простого в сравнении с квадратичными критериями (см. [16]) и позволяющего решать задачи (25) редукцией к задачам линейного программирования.

6. Заключение. В работе для ряда актуальных задач оптимального управления обсуждается применение принципа управления в режиме реального времени. Приведены алгоритмы работы оптимальных регуляторов, реализующих оптимальные обратные связи в реальном времени. Алгоритмы используют методы решения задач оптимального управления, в основе которых лежат специальная параметризация и быстрые алгоритмы коррекции оптимальных программ (см. [4]). Эффективность методов продемонстрирована на примерах управления системой шестого порядка.

Отметим, что идеи управления в реальном времени развиваются в $[11,14]$ для задач сближения и наведения. Идеи оптимального децентрализованного управления могут найти применение при 
решении задач синхронизации, а также допускают развитие на случай оптимального управления по несовершенным измерениям, как в разделе 3 , и децентрализованной стабилизации групп динамических объектов.

\section{СПИСОК ЛИТЕРАТУРЫ}

1. Балашевич H. В. О стабилизации динамических систем ограниченными обратными связями// Тр. Ин-та мат. НАН Беларуси. - 1999. - № 3. - С. 38-45.

2. Габасов P., Кириллова Ф. М. Оптимальное управление в реальном времени многомерным динамическим объектом// Автомат. телемех. - 2015. - № 1. - С. 121-135.

3. Габасов Р., Кириллова Ф. М., Дмитрук Н. М. Синтез оптимальных систем - управление в реальном времени// в кн.: Тр. Междунар. конф. «Динамика систем и процессы управления», посв. 90-летию академика Н. Н. Красовского, 2015. - С. 208-219.

4. Габасов P., Кириллова Ф. М., Павленок Н. С. Оптимальное управление динамическим объектом по совершенным измерениям его состояний// Докл. РАН. - 2012. - 444, № 4. - С. 371-375.

5. Габасов Р., Кириллова Ф. М., Во Тхи Танъ Ха Применение численных методов линейного программирования для управления в реальном времени линейным динамическим объектом// Ж. вычисл. мат. мат. физ. - 2016. - 56, № 3. - С. 394-408.

6. Габасов P., Кириллова Ф. М. О некоторых проблемах оптимального управления в реальном времени линейными стационарными системами// Изв. Иркутск. ун-та. Сер. мат. - 2019. - 27. - С. 15-27.

7. Габасов P., Кириллова Ф. М., Поясок Е. И. Оптимальное управление по несовершенным измерениям входных и выходных сигналов динамическим объектом с множественной неопределенностью в начальном состоянии // Ж. вычисл. мат. мат. физ. - 2012. - 52, № 7. - С. 1215-1230..

8. Габасов Р., Кириллова Ф. М., Поясок Е. И. Оптимальное наблюдение в реальном времени линейного динамического объекта// Докл. РАН. - 2013. - 448, № 3. - С. 145-148.

9. Габасов Р., Кириллова Ф. М., Дмитрук Н. М. Оптимальное децентрализованное управление динамическими системами в условиях неопределенности// Ж. вычисл. мат. мат. физ. - 2011. - 51, № 7. C. $1209-1227$.

10. Габасов P., Кириллова Ф. М., Дмитрук Н. М. Децентрализованное управление в линейно квадратичной задаче при наличии запаздывания в информационном канале// Докл. НАН Беларуси. - 2016. 60, № 3. - C. 18-24.

11. Габасов Р., Дмитрук Н. М., Кириллова Ф. М., Поясок Е. И. Задачи оптимального управления с подвижной целью// Тр. Ин-та мат. мех. УрО РАН. - 2010. - 16, № 5. - С. 16-21.

12. Дмитрук H. М. Оптимальное управление взаимосвязанными объектами// Динамика систем и процессы управл. - 2015. - C. 147-154.

13. Christofides P. D., Scattolini R., de la Pena D. M., Liu J. Distributed model predictive control: A tutorial review and future research directions// Comput. Chem. Eng. — 2013. — 51. — P. 21-41.

14. Gabasov R., Dmitruk N. M., Kirillova F. M. On optimal control of an object at its approaching to moving target under uncertainty// Appl. Comput. Math. — 2013. — 12, № 2. — P. 152-167.

15. Jia D., Krogh B. Min-max feedback model predictive control for distributed control with communication// Proc. 2002 Am. Control Conf. — 2002. - 6. - P. 4507-4512.

16. Rawlings J.B., Mayne D.Q. Model predictive control: Theory and design. - Nob Hill Publ., 2009.

Габасов Рафаил

Белорусский государственный университет, Минск, Республика Беларусь

Дмитрук Наталия Михайловна

Белорусский государственный университет, Минск, Республика Беларусь

E-mail: dmitrukn@bsu. by

Кириллова Фаина Михайловна

Институт математики НАН Беларуси, Минск, Республика Беларусь

E-mail: kirillova.f@yandex.by 